\title{
Assessment of anthropogenic Cesium-137 in soil of Islamabad capital territory and its suburbs, Pakistan
}

\author{
I.U. Khan ${ }^{1}$, W. Sun ${ }^{1}$, E. Lewis ${ }^{2}$ \\ ${ }^{1}$ Key Lab of In-fiber Integrated Optics, Ministry Education of China, Harbin Engineering University, Harbin, China \\ ${ }^{2}$ Optical Fibre Sensors Research Centre, University of Limerick, Castletroy, Limerick, Ireland
}

\section{- Original article}

*Corresponding authors:
Dr. Weimin Sun,
E-mail:
$\quad$ sunweimin@hrbeu.edu.cn
Revised: March 2020
Accepted: May 2020
Int. J. Radiat. Res., April 2021;
19(2): 325-332
DOI: $10.29252 /$ ijrr.19.2.325

\begin{abstract}
Background: Cesium-137 is one of those man-made radioactive nuclides that results from human activities including open air nuclear weapons testing, nuclear accidents, operation of nuclear reactors and has a relative long halflife, higher water solubility, high fission yield, relatively high volatility and a close physic-chemical similarity to potassium that makes it a dangerous radioactive element in the human environment and is a hazardous byproduct of nuclear fission. Cesium-137 being a $y$-emitter is also a potential carcinogen. Materials and Methods: A total of 100 soil samples were collected from various sites in Islamabad and its suburbs for the assessment of the presence of Cesium-137 in the environment. Spectroscopy of the collected samples for determination of aforementioned radionuclide was performed using a computer based High Purity Germanium (HPGe) detector. Results: The highest value of Cesium-137 was found to be $57.0 \pm 4.2 \mathrm{~Bq} / \mathrm{kg}$ in samples collected from Nilore, a suburb of Islamabad. Conclusion: The presence of Cesium-137 in all samples is clear evidence that the studied area is contaminated either due to past fallout or ongoing nuclear activities in this area. Cesium-137 was found in higher concentration for this area than the reported values in literature for the rest of country, but not to an alarming level. However, local concerned authorities must pay attention to avoid the development of possible future health hazards to the residents of this area.
\end{abstract}

Keywords: Radioactivity, cesium-137, gamma spectroscopy, Islamabad capital territory.

\section{INTRODUCTION}

The global environment has been and continues to be subjected to exposure to ionizing radiation. This implies that almost all living organisms including human beings are exposed to and accumulate dose from varying amounts of radiation from both natural and anthropogenic sources. These exposures induce various biological effects by altering the structure of cells and lead to damage of DNA (deoxyribonucleic acid). DNA damage results in potential mutation of genes, chromosomal abnormality and malfunction or death of the cells (1). Long term exposure of humans to ionizing radiation may also result in chronic lung disease, cataract, anemia, necrosis of the mouth, acute leucopoenia, lung cancer and leukemia (2-5). Cancer is still one of the deleterious effects caused by radiation from primordial as well as anthropogenic sources.

Anthropogenic radioactivity emerges purely from human activities on this planet, which includes testing of nuclear weapons, operation of nuclear power reactors and nuclear medicine (6). The assessment of artificial radioactivity has drawn much global attention from researchers especially following the well-publicized nuclear reactor incidents in Japan (Fukushima, 2011) and Ukraine (Chernobyl, 1986) (7).

Strontium-90 and Cesium-137 are considered foremost among all of the fall out radioactive constituent due to their high toxicity, long half-lives, and higher uptake by bio systems (8). Cesium-137 is perhaps the most significant fission by-product due to volatility, with a half-life of more than thirty years (30.17 years). It has since been distributed throughout the Earth's atmosphere due to global 
atmospheric weather patterns. Its water solubility is another measurable factor, which plays an important role in defining its concentration in the environment (9). Historical atmospheric nuclear weapons testing and other detonations constitutes a significant source of Cesium-137 is of these $60 \%$ of the collective effective dose equivalent from external radiation resulted from ground based testing (10). It also gets into our environment via routine operation of nuclear reactors for power generation and military purposes, radioactive fuel reprocessing plants, and sites for nuclear waste disposal (8). Therefore, analyzing the concentration of Cesium-137 in soil is essential valuable indicator of the environmental effect of radioactivity level, as soil is one of the most important materials which are used for many purposes in modern society (11).

Cesium-137 can enter the food chain either by direct accumulation on exposed plants or by its solubility in water through the uptake of the plant's roots system. Cesium isotopes are very similar in chemical and physical properties to Potassium and are therefore easily accumulated in plant and animal tissues. Therefore, soil composition (chemistry), as well as retention of the isotope in the top surface soil structure, plays an important role (12). A large number of global monitoring bodies, researchers and scientists have expressed concern for the level of radioactivity from non-natural sources, especially the anthropogenic fission by-product (Cs-137) (13). The potential environmental impacts of the Fukushima (2011) and Chernobyl (1986) nuclear reactor incidents are reviewed in reference (14).

Environmental samples including soil, air, water and, vegetation have been assessed for natural as well as artificial radioactivity by various researchers from across the world (15, 16, 25-34, 17-24). The present work constitutes one of these ongoing investigations and is centered on Pakistan.. The mountainous region of Islamabad in Pakistan is a potential geographical region with higher concentrations of anthropogenic radio-active fall-out and therefore, it is desirable to perform a gamma survey of this area. This is the first time that such a survey has been undertaken. This article mainly deals with the quantitative assessment of radioactivity concentration of Cs-137 in clay samples obtained from various sectors of Islamabad and its suburb particularly Nilore.

The results obtained are compared with the data available in the literature for the Islamic Republic of Pakistan as well as other countries around the globe. Radioactivity assessment of Cesium-137 in soil of residential as well as cultivated suburbs of Islamabad is important as it is one of the major sources of radionuclide entrainment into the food chain. The main aim of this study is to undertake a survey for the existence of cesium-137 radioactive nuclides in the soil and to establish a reference line for further and future work on radioactivity concentration in this locality.

\section{MATERIALS AND METHODS}

\section{Description of study sites}

Islamabad is Pakistan's federal capital city envisaged in 1960 as the new capital of the country, situated geographically $\left(33.49^{\circ} \mathrm{N}\right.$, $72.24^{\circ} \mathrm{E}$ ) at the edge of the Pothohar Plateau at the foot of the Margalla Hills in the northeast of the country with varying altitude ranging from 457 to $610 \mathrm{~m}$. Its climate is subtropical humid. This kind of climate is characterized by hot summers with a monsoon followed by cold winters. The average annual rainfall is about 41 inches with over fifty percent (50\%) precipitation taking place due to the monsoon system. The location of Islamabad makes it a gateway to both Punjab and Khyber Pakhtunkhwa, as it falls at the intersection of the two provinces of Islamic Republic of Pakistan. This well planned city of the country occupies an area of $906.50 \mathrm{Km}^{2}$ with a population greater than 2.0 million according to the 2017 census conducted by the Pakistan Bureau of statistics. For administrative purposes, the city is divided into sectors, which can be identified by an English alphabet with a number shown in $f$ igure 1 and each sector covers an area of approximately $2 \mathrm{~km} \times 2 \mathrm{~km}$.

\section{Sample collection}

A total of 100 soil samples (IS-1 to NS-100) from Islamabad various sectors of capital territory and its suburbs were collected as shown in figure1. The adopted strategy for sampling was according to the standard method outlined in IAEA (International Atomic Energy Agency) report No. 295 (35). An area of approximately $1 \mathrm{~m} \times 1 \mathrm{~m}$ of undisturbed, open and relatively level ground was marked for collection of each sample by removing the surface layer contain gravels, stone pieces, grass and its roots. The samples were taken to a depth

Int. J. Radiat. Res., Vol. 19 No. 2, April 2021 


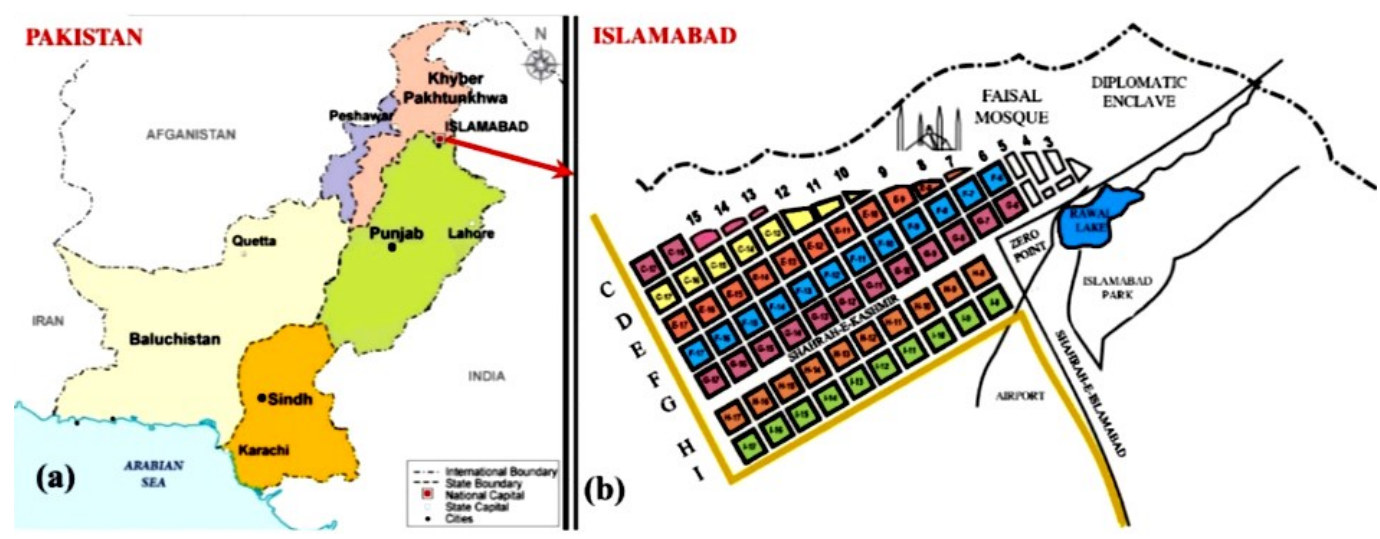

Figure 1. (a) Islamabad location on Pakistan's map and (b) Sectorial map of Islamabad.

of 5-15 centimeters from each corner and one from center of the marked area of $1 \mathrm{~m}^{2}$ using a standard coring tool. A minimum distance of two kilometers was maintained between any two sampling sites. The collected soil was then mixed well to obtain a representative sample of the specified location each of weight $2.0 \mathrm{Kg}$. The geographical location (Latitude, Longitude) of each sample was recorded using a Garmin eTrex 309x GPS (Global Positioning System) unit. The samples were placed in previously unused properly polyethylene bags labeled with the site codes and were subsequently transported to a laboratory of Radiation Surveillance Center (RSC), Islamabad for further processing.

\section{Sample preparation}

The collected soil samples were initially spread on a plastic mat and allowed to dry at room temperature for a period of one week to avoid any loss of radioactive content. Constant weight was achieved as each sample was further dried overnight in an electric furnace at a temperature of $110 \pm 5{ }^{\circ} \mathrm{C}$. After that the dried samples were then ground, pulverized and powdered by some mechanical treatment and passed through a sieve having mesh of size 0.2 centimeters. These prepared samples each weighing $0.5 \mathrm{Kg}$ were then sealed in air-tight Marinelli beakers keeping its geometrical dimension exactly identical to the International Atomic Energy Agency (IAEA) provided reference soil-375. The samples geometrical dimensions were identical to the calibration requirement (36).

\section{Spectroscopy for gamma activity}

A high resolution activity measuring system comprising a low energy high-purity germanium semiconductor (HPGe) detector (Model: GC3020 Canberra, USA) was used for radiometric assessment of the collected samples. The detector has a relative efficiency of $30 \%$ with an active volume $180 \mathrm{~mm}^{3}$.The detector was housed in a lead shielding of well-type (thickness $5-15 \mathrm{~cm}$ ) with a fixed bottom and removable cover for the reduction of background radiation from its surrounding environment. Calibration of this measuring system was performed using a reference material (Soil-375) obtained from the International Atomic Energy Agency (IAEA, Vienna, Austria) in order to determine the activity of the Cesium-137 $\gamma$-ray in the collected soil samples (36). The detector is subjected to a high operating voltage of $3 \mathrm{KV}$ via a preamplifier, which was then coupled to a PC based multichannel analyzer (Accuspec-A, Canberra) having 8192 channels through an analogue to digital converter (ADC) for the acquisition of the processed data. The activities concentrations and corresponding uncertainties were estimated according to statistical uncertainty of peak areas given by commercially available (Genie-2000 Canberra, USA) software along with Excel (Microsoft Corporation, Redmond, WA). The detector was continuously cooled using liquid nitrogen in order to minimize the detector thermal noise. The system energy resolution at $1332.5 \mathrm{keV}$ from ${ }^{60} \mathrm{Co}$ is $2 \mathrm{KeV}$ FWHM. The spectrum for each of $0.5 \mathrm{~kg}$ dried soil sample 
was acquired continuously for about 18 hours to achieve adequate counts at the anticipated peaks. The lower Limits of Detection (LLD) for Cesium-137 was obtained as $1.24 \mathrm{~Bq} / \mathrm{kg}$ with a 95\% confidence level. The activities of Cesium-137 were determined from the gamma ray lines of energy $661.7 \mathrm{KeV}$ present in the acquired spectrum of energies for the analyzed soil samples. Signals from the surrounding environment of the laboratory were also continuously recorded and were subtracted from each measurement. The activity mass concentration in $\mathrm{Bq} / \mathrm{kg}$ of $\mathrm{Cs}-137$ was calculated using equation 1 as follows $(20,21)$.

$$
A_{s}=\frac{C_{S}-C_{B}}{E_{\gamma} \times P_{\gamma} \times T \times M}
$$

Where,

$\mathrm{A}_{\mathrm{s}}=$ activity mass concentration

$\mathrm{C}_{\mathrm{s}}=$ Counts/second for sample

$\mathrm{C}_{\mathrm{B}}=$ Counts/second for background

$\mathrm{E}_{\gamma}=$ Detector efficiency for gamma ray

$\mathrm{P}_{\gamma}=$ Probability of gamma emission

$\mathrm{T}=$ Counting time

$M=$ Mass of sample

\section{RESULTS AND DISCUSSION}

The assessment of radioactivity concentration of anthropogenic Cesium-137 in soil samples collected from various sectors of Islamabad capital territory and its suburbs was conducted using a direct $\gamma$-ray spectrometry technique. The resulting values are reported in Becquerel per kilogram (Bq/kg) for collected samples on a dry weight basis. Activity concentration with plus minus counting uncertainty was found to be in the range of $8.8 \pm 2.8(\mathrm{~Bq} / \mathrm{kg})$ to $57.0 \pm 4.2(\mathrm{~Bq} / \mathrm{kg})$ as shown in table 1.

Results indicate the variation of Cesium-137 activity concentration from sample to sample. This variation may be caused by various factors including variations in local soil characteristics of the sampling site and metrological conditions. The latter is dominated by the amount of rainfall in the preceding few days, which plays a significant role in the deposition of the radio-nucleotide in the soil. Area topography is another factor affecting its accumulation. However this variation in activity concentration of radiocesium is considered as insignificant keeping in view the large geographic and geological diversification. The pre-mentioned factors may be accountable for the variation in activity level of Cesium-137 in the soil of this territory.

The results of the present work were compared to the available data in the literature for various parts of the country as well as some other countries around the globe as shown in table 2. It is evident from table 2 that the maximum value of radioactivity concentration of Cesium-137 in soil of Islamabad capital territory is comparatively higher than the maximum value reported for Kohistan (37), Jhangar Valley (38), Peshawar and Nowshera (39), Murree (40), Jhelum , Attock and, Chakwal (41), Southern Punjab (42) and Mirpur, Azad Kashmir, Pakistan (43) and some other environs of the world including Tehran (Iran) (44), Tongliao \& Bayanwula (China) $(45,46)$, İkizdere Valley (Turkey) (47). The elevated concentrations of Cesium-137 in soil samples collected from suburbs of Islamabad i.e. Nilore may either be due to the operation of a nearby nuclear research reactor under the control of Pakistan Atomic Energy Commission (PAEC) without any interruption for the last few decades or the area may have experienced high past fallout. The data of this investigation is in close agreement with the maximum values reported for Louisiana (USA) (39) and Vojvodina (Serbia) (48) but significantly less than data available for Syria (49) as well as the Leningrad Region (Russia) (50), where Cesium-137 is deposited with higher concentration in surrounding environment after the occurrence of the Chernobyl nuclear reactor incident near Russia. 
Table 1. Activity concentration ( \pm counting uncertainty) of anthropogenic Cesium-137 in soil samples collected from Islamabad and its suburbs.

\begin{tabular}{|c|c|c|c|c|c|c|c|}
\hline Sectors & \begin{tabular}{|c|} 
Sample \\
ID
\end{tabular} & Coordinates & $\begin{array}{l}\text { Activity } \\
\text { (Bq/kg) }\end{array}$ & Sectors & $\begin{array}{c}\text { Sample } \\
\text { ID }\end{array}$ & Coordinates & $\begin{array}{l}\text { Activity } \\
\text { (Bq/kg) }\end{array}$ \\
\hline \multirow{5}{*}{ C } & IS-1 & $33^{\circ} 42^{\prime} 44.43^{\prime \prime} \mathrm{N}, 72^{\circ} 55^{\prime} 11.42^{\prime \prime} \mathrm{E}$ & $13.1 \pm 3.1$ & \multirow{6}{*}{ H } & IS-51 & $33^{\circ} 38^{\prime} 46.92^{\prime \prime} \mathrm{N}, 72^{\circ} 59^{\prime} 53.18^{\prime \prime E}$ & $26.8 \pm 3.1$ \\
\hline & IS-2 & $33^{\circ} 42^{\prime} 20.92^{\prime \prime} \mathrm{N}, 72^{\circ} 55^{\prime} 30.90^{\prime \prime E}$ & $16.2 \pm 3.4$ & & IS-52 & $33^{\circ} 38^{\prime} 41.02^{\prime \prime} \mathrm{N}, 72^{\circ} 58^{\prime} 32.91^{\prime \prime} \mathrm{E}$ & $26.1 \pm 3.3$ \\
\hline & IS-3 & $33^{\circ} 42^{\prime} 31.97^{\prime \prime} \mathrm{N}, 72^{\circ} 56^{\prime} 8.70^{\prime \prime} \mathrm{E}$ & $18.3 \pm 2.9$ & & IS-53 & $33^{\circ} 37^{\prime} 35.77^{\prime \prime} \mathrm{N}, 72^{\circ} 58^{\prime} 15.76^{\prime \prime E}$ & $33.3 \pm 2.9$ \\
\hline & IS-4 & $33^{\circ} 43^{\prime} 0.39^{\prime \prime} \mathrm{N}, 72^{\circ} 56^{\prime} 20.38^{\prime \prime} \mathrm{E}$ & $20.4 \pm 3.7$ & & IS-54 & $33^{\circ} 38^{\prime} 56.61^{\prime \prime} \mathrm{N}, 72^{\circ} 59^{\prime} 37.49^{\prime \prime E}$ & $30.0 \pm 3.7$ \\
\hline & IS-5 & $33^{\circ} 42^{\prime} 34.45^{\prime \prime} \mathrm{N}, 33^{\circ} 42^{\prime} 34.45^{\prime \prime} \mathrm{N}$ & $24.6 \pm 3.5$ & & IS-55 & $33^{\circ} 37^{\prime} 47.86^{\prime \prime} \mathrm{N}, 72^{\circ} 58^{\prime} 42.43^{\prime \prime} \mathrm{E}$ & $32.5 \pm 2.8$ \\
\hline \multirow{7}{*}{ D } & IS-6 & $33^{\circ} 42^{\prime} 29.95^{\prime \prime} \mathrm{N}, 72^{\circ} 57^{\prime} 11.76^{\prime \prime} \mathrm{E}$ & $12.3 \pm 2.9$ & & IS-56 & $33^{\circ} 37^{\prime} 56.05^{\prime \prime} \mathrm{N}, 72^{\circ} 58^{\prime} 12.15^{\prime \prime} \mathrm{E}$ & $33.4 \pm 3.6$ \\
\hline & IS-7 & $33^{\circ} 42^{\prime} 3.52^{\prime \prime} \mathrm{N}, 72^{\circ} 57^{\prime} 13.91^{\prime \prime} \mathrm{E}$ & $15.7 \pm 3.5$ & \multirow{10}{*}{ I } & IS-57 & $33^{\circ} 40^{\prime} 2.19^{\prime \prime} \mathrm{N}, 73^{\circ} 4^{\prime} 20.99^{\prime \prime} \mathrm{E}$ & $11.5 \pm 3.0$ \\
\hline & IS-8 & $33^{\circ} 41^{\prime} 53.32^{\prime \prime} \mathrm{N}, 72^{\circ} 56^{\prime} 35.86^{\prime \prime} \mathrm{E}$ & $19.7 \pm 3.1$ & & IS-58 & $33^{\circ} 39^{\prime} 12.36^{\prime \prime} \mathrm{N}, 73^{\circ} 3^{\prime} 30.02^{\prime \prime} \mathrm{E}$ & $14.7 \pm 2.9$ \\
\hline & IS-9 & $33^{\circ} 42^{\prime} 9.81^{\prime \prime} \mathrm{N}, 72^{\circ} 56^{\prime} 26.03^{\prime \prime E}$ & $22.8 \pm 3.6$ & & IS-59 & $33^{\circ} 38^{\prime} 56.57^{\prime \prime} \mathrm{N}, 73^{\circ} 2^{\prime} 29.99^{\prime \prime E}$ & $16.6 \pm 3.1$ \\
\hline & IS-10 & $33^{\circ} 42^{\prime} 24.67^{\prime \prime} \mathrm{N}, 72^{\circ} 56^{\prime} 50.17^{\prime \prime} \mathrm{E}$ & $25.6 \pm 3.2$ & & IS-60 & $33^{\circ} 38^{\prime} 14.47^{\prime \prime} \mathrm{N}, 73^{\circ} 1^{\prime} 14.94^{\prime \prime E}$ & $19.3 \pm 2.8$ \\
\hline & IS-11 & $33^{\circ} 42^{\prime} 4.02^{\prime \prime} \mathrm{N}, 72^{\circ} 57^{\prime} 33.26^{\prime \prime} \mathrm{E}$ & $26.9 \pm 3.4$ & & IS-61 & $33^{\circ} 35^{\prime} 51.95^{\prime \prime} \mathrm{N}, 73^{\circ} 13^{\prime} 0.64^{\prime \prime} \mathrm{E}$ & $23.7 \pm 3.2$ \\
\hline & IS-12 & $33^{\circ} 42^{\prime} 15.48^{\prime \prime} \mathrm{N}, 72^{\circ} 57^{\prime} 9.91^{\prime \prime E}$ & $28.1 \pm 2.9$ & & IS-62 & $33^{\circ} 37^{\prime} 33.01^{\prime \prime} \mathrm{N}, 72^{\circ} 59^{\prime} 46.69^{\prime \prime} \mathrm{E}$ & $26.8 \pm 3.4$ \\
\hline \multirow{11}{*}{ E } & IS-13 & $33^{\circ} 43^{\prime} 38.54^{\prime \prime N}, 73^{\circ} 3^{\prime} 5.17^{\prime \prime E}$ & $12.5 \pm 3.5$ & & IS-63 & $33^{\circ} 36^{\prime} 28.87^{\prime \prime} \mathrm{N}, 72^{\circ} 58^{\prime} 0.52^{\prime \prime} \mathrm{E}$ & $28.6 \pm 3.1$ \\
\hline & IS-14 & $33^{\circ} 43^{\prime} 22.52^{\prime \prime} \mathrm{N}, 73^{\circ} 1^{\prime} 57.18^{\prime \prime} \mathrm{E}$ & $14.0 \pm 3.0$ & & IS-64 & $33^{\circ} 35^{\prime} 57.02^{\prime \prime} \mathrm{N}, 73^{\circ} 12^{\prime} 58.67^{\prime \prime} \mathrm{E}$ & $30.7 \pm 2.8$ \\
\hline & IS-15 & $33^{\circ} 42^{\prime} 54.61^{\prime \prime} \mathrm{N}, 73^{\circ} 1^{\prime} 14.88^{\prime \prime} \mathrm{E}$ & $16.8 \pm 2.9$ & & IS-65 & $33^{\circ} 35^{\prime} 50.68^{\prime \prime} \mathrm{N}, 72^{\circ} 56^{\prime} 10.89^{\prime \prime} \mathrm{E}$ & $31.0 \pm 3.2$ \\
\hline & IS-16 & $33^{\circ} 42^{\prime} 44.25^{\prime \prime} \mathrm{N}, 73^{\circ} 0^{\prime} 48.92^{\prime \prime} \mathrm{E}$ & $19.3 \pm 3.6$ & & IS-66 & $33^{\circ} 35^{\prime} 36.12^{\prime \prime} \mathrm{N}, 73^{\circ} 12^{\prime} 46.80^{\prime \prime} \mathrm{E}$ & $33.5 \pm 2.9$ \\
\hline & IS-17 & $33^{\circ} 42^{\prime} 25.33^{\prime \prime} \mathrm{N}, 73^{\circ} 0{ }^{\prime} 19.75^{\prime \prime E}$ & $23.1 \pm 2.8$ & \multirow{34}{*}{ Nilore } & NS-67 & $33^{\circ} 39^{\prime} 8.22^{\prime \prime} \mathrm{N}, 73^{\circ} 14^{\prime} 6.01^{\prime \prime E}$ & $41.8 \pm 4.1$ \\
\hline & IS-18 & $33^{\circ} 42^{\prime} 20.99^{\prime \prime} \mathrm{N}, 72^{\circ} 59^{\prime} 26.15^{\prime \prime} \mathrm{E}$ & $22.4 \pm 3.2$ & & NS-68 & $33^{\circ} 39^{\prime} 10.47^{\prime \prime} \mathrm{N}, 73^{\circ} 14^{\prime} 29.03^{\prime \prime} \mathrm{E}$ & $50.4 \pm 3.8$ \\
\hline & IS-19 & $33^{\circ} 42^{\prime} 4.69^{\prime \prime} \mathrm{N}, 72^{\circ} 58^{\prime} 55.49^{\prime \prime} \mathrm{E}$ & $27.2 \pm 3.7$ & & NS-69 & $33^{\circ} 39^{\prime} 0.64^{\prime \prime N}, 73^{\circ} 14^{\prime} 48.49^{\prime \prime E}$ & $57.0 \pm 4.2$ \\
\hline & IS-20 & $33^{\circ} 41^{\prime} 52.08^{\prime \prime} \mathrm{N}, 72^{\circ} 58^{\prime} 33.78^{\prime \prime} \mathrm{E}$ & $29.2 \pm 3.8$ & & NS-70 & $33^{\circ} 38^{\prime} 47.50^{\prime \prime} \mathrm{N}, 73^{\circ} 15^{\prime} 22.54^{\prime \prime} \mathrm{E}$ & $44.8 \pm 2.8$ \\
\hline & IS-21 & "N, 7253'18.88"E & $30.9 \pm 3.9$ & & NS-71 & $33^{\circ} 38^{\prime} 51.39^{\prime \prime} \mathrm{N}, 73^{\circ} 15^{\prime} 31.29^{\prime \prime E}$ & $55.1 \pm 3.7$ \\
\hline & IS-22 & $33^{\circ} 39^{\prime} 11.10^{\prime \prime} \mathrm{N}, 72^{\circ} 52^{\prime} 38.32^{\prime \prime} \mathrm{E}$ & $29.4 \pm 3.5$ & & NS-72 & $33^{\circ} 38^{\prime} 54.62^{\prime \prime} \mathrm{N}, 73^{\circ} 15^{\prime} 45.18^{\prime \prime} \mathrm{E}$ & $51.6 \pm 3.2$ \\
\hline & IS-23 & $33^{\circ} 38^{\prime} 0.68^{\prime \prime} \mathrm{N}, 72^{\circ} 51^{\prime} 6.15^{\prime \prime} \mathrm{E}$ & $31.3 \pm 2.9$ & & NS-73 & $33^{\circ} 39^{\prime} 7.66^{\prime \prime} \mathrm{N}, 73^{\circ} 15^{\prime} 58.83^{\prime \prime} \mathrm{E}$ & $49.3 \pm 3.9$ \\
\hline \multirow{11}{*}{$\mathbf{F}$} & IS-24 & $33^{\circ} 43^{\prime} 31.56^{\prime \prime} \mathrm{N}, 73^{\circ} 4^{\prime} 43.14^{\prime \prime} \mathrm{E}$ & $11.3 \pm 3.6$ & & NS-74 & $33^{\circ} 39^{\prime} 0.80^{\prime \prime} \mathrm{N}, 73^{\circ} 16^{\prime} 7.99^{\prime \prime} \mathrm{E}$ & $45.7 \pm 3.0$ \\
\hline & IS-25 & $33^{\circ} 43^{\prime} 50.76^{\prime \prime} \mathrm{N}, 73^{\circ} 3^{\prime} 54.14^{\prime \prime E}$ & $12.0 \pm 2.8$ & & NS-75 & $33^{\circ} 38^{\prime} 53.44^{\prime \prime} \mathrm{N}, 73^{\circ} 16^{\prime} 53.76^{\prime \prime E}$ & $48.6 \pm 3.2$ \\
\hline & IS-26 & $33^{\circ} 42^{\prime} 48.47^{\prime \prime} \mathrm{N}, 73^{\circ} 1^{\prime} 56.54^{\prime \prime E}$ & $16.6 \pm 3.7$ & & NS-76 & $33^{\circ} 39^{\prime} 1.84^{\prime \prime} \mathrm{N}, 73^{\circ} 17^{\prime} 1.29^{\prime \prime} \mathrm{E}$ & $56.3 \pm 3.8$ \\
\hline & IS-27 & $33^{\circ} 42^{\prime} 12.70^{\prime \prime} \mathrm{N}, 73^{\circ} 2^{\prime} 15.14^{\prime \prime} \mathrm{E}$ & $18.5 \pm 2.9$ & & NS-77 & $33^{\circ} 39^{\prime} 6.53^{\prime \prime} \mathrm{N}, 73^{\circ} 16^{\prime} 52.99^{\prime \prime} \mathrm{E}$ & $50.4 \pm 3.0$ \\
\hline & IS-28 & $33^{\circ} 42^{\prime} 15.04^{\prime \prime} \mathrm{N}, 73^{\circ} 1^{\prime} 40.82^{\prime \prime} \mathrm{E}$ & $19.6 \pm 3.2$ & & NS-78 & $33^{\circ} 39^{\prime} 13.65^{\prime \prime} \mathrm{N}, 73^{\circ} 16^{\prime} 43.43^{\prime \prime} \mathrm{E}$ & $43.8 \pm 2.9$ \\
\hline & IS-29 & $33^{\circ} 42^{\prime} 2.68^{\prime \prime} \mathrm{N}, 73^{\circ} 0^{\prime} 29.41^{\prime \prime E}$ & $22.8 \pm 3.0$ & & NS-79 & $33^{\circ} 39^{\prime} 20.14^{\prime \prime} \mathrm{N}, 73^{\circ} 16^{\prime} 43.01^{\prime \prime} \mathrm{E}$ & $44.5 \pm 3.6$ \\
\hline & IS-30 & $33^{\circ} 40^{\prime} 32.86^{\prime \prime} \mathrm{N}, 72^{\circ} 59^{\prime} 8.19^{\prime \prime E}$ & $21.7 \pm 3.6$ & & NS-80 & $33^{\circ} 39^{\prime} 23.32^{\prime \prime} \mathrm{N}, 73^{\circ} 16^{\prime} 42.66^{\prime \prime E}$ & $49.5 \pm 3.4$ \\
\hline & IS-31 & $33^{\circ} 41^{\prime} 4.30^{\prime \prime} \mathrm{N}, 72^{\circ} 58^{\prime} 47.71^{\prime \prime} \mathrm{E}$ & $22.5 \pm 3.1$ & & NS-81 & $33^{\circ} 39^{\prime} 27.13^{\prime \prime} \mathrm{N}, 73^{\circ} 16^{\prime} 34.94^{\prime \prime} \mathrm{E}$ & $53.1 \pm 3.2$ \\
\hline & IS-32 & $33^{\circ} 37^{\prime} 52.66^{\prime \prime} \mathrm{N}, 72^{\circ} 52^{\prime} 25.43^{\prime \prime} \mathrm{E}$ & $26.3 \pm 2.9$ & & NS-82 & $33^{\circ} 39^{\prime} 29.09^{\prime \prime} \mathrm{N}, 73^{\circ} 16^{\prime} 29.47^{\prime \prime} \mathrm{E}$ & $42.9 \pm 3.4$ \\
\hline & IS-33 & $33^{\circ} 38^{\prime} 19.36^{\prime \prime} \mathrm{N}, 72^{\circ} 53^{\prime} 15.40^{\prime \prime} \mathrm{E}$ & $30.4 \pm 3.3$ & & NS-83 & $33^{\circ} 39^{\prime} 31.52^{\prime \prime} \mathrm{N}, 73^{\circ} 16^{\prime} 24.18^{\prime \prime E}$ & $46.7 \pm 3.5$ \\
\hline & IS-34 & $33^{\circ} 37^{\prime} 26.12^{\prime \prime} \mathrm{N}, 72^{\circ} 52^{\prime} 35.69^{\prime \prime} \mathrm{E}$ & $34.1 \pm 3.2$ & & NS-84 & $33^{\circ} 39^{\prime} 32.47^{\prime \prime} \mathrm{N}, 73^{\circ} 16^{\prime} 17.07^{\prime \prime} \mathrm{E}$ & $41.1 \pm 2.8$ \\
\hline \multirow{16}{*}{ G } & IS-35 & $33^{\circ} 43^{\prime} 12.71^{\prime \prime} \mathrm{N}, 73^{\circ} 5^{\prime} 57.21^{\prime \prime} \mathrm{E}$ & $8.8 \pm 2.8$ & & NS-85 & $33^{\circ} 39^{\prime} 37.89^{\prime \prime} \mathrm{N}, 73^{\circ} 16^{\prime} 10.18^{\prime \prime} \mathrm{E}$ & $40.9 \pm 3.0$ \\
\hline & IS-36 & $33^{\circ} 42^{\prime} 48.75^{\prime \prime} \mathrm{N}, 73^{\circ} 5^{\prime} 20.64^{\prime \prime E}$ & $12.0 \pm 3.1$ & & NS-86 & $33^{\circ} 39^{\prime} 40.79^{\prime \prime} \mathrm{N}, 73^{\circ} 16^{\prime} 4.41^{\prime \prime} \mathrm{E}$ & $56.4 \pm 3.1$ \\
\hline & IS-37 & $33^{\circ} 42^{\prime} 32.07^{\prime \prime} \mathrm{N}, 73^{\circ} 4^{\prime} 24.41^{\prime \prime} \mathrm{E}$ & $16.7 \pm 3.6$ & & NS-87 & $33^{\circ} 39^{\prime} 39.16^{\prime \prime} \mathrm{N}, 73^{\circ} 15^{\prime} 54.52^{\prime \prime} \mathrm{E}$ & $55.9 \pm 3.9$ \\
\hline & IS-38 & $33^{\circ} 42^{\prime} 2.95^{\prime \prime} \mathrm{N}, 73^{\circ} 3^{\prime} 23.76^{\prime \prime E}$ & $20.2 \pm 2.9$ & & NS-88 & $33^{\circ} 39^{\prime} 39.28^{\prime \prime} \mathrm{N}, 73^{\circ} 15^{\prime} 12.91^{\prime \prime E}$ & $50.1 \pm 3.0$ \\
\hline & IS-39 & $33^{\circ} 41^{\prime} 8.96^{\prime \prime} \mathrm{N}, 73^{\circ} 2^{\prime} 0.68^{\prime \prime} \mathrm{E}$ & $26.3 \pm 3.2$ & & NS-89 & $33^{\circ} 39^{\prime} 39.06^{\prime \prime} \mathrm{N}, 73^{\circ} 15^{\prime} 4.85^{\prime \prime} \mathrm{E}$ & $54.5 \pm 3.7$ \\
\hline & IS-40 & $33^{\circ} 40^{\prime} 31.83^{\prime \prime} \mathrm{N}, 73^{\circ} 1^{\prime} 18.71^{\prime \prime E}$ & $29.7 \pm 3.4$ & & NS-90 & $33^{\circ} 39^{\prime} 35.81^{\prime \prime} \mathrm{N}, 73^{\circ} 15^{\prime} 0.71^{\prime \prime} \mathrm{E}$ & $53.4 \pm 3.1$ \\
\hline & IS-41 & $33^{\circ} 40^{\prime} 2.53^{\prime \prime} \mathrm{N}, 73^{\circ} 0^{\prime} 18.62^{\prime \prime E}$ & $19.3 \pm 3.7$ & & NS-91 & $33^{\circ} 39^{\prime} 30.65^{\prime \prime} \mathrm{N}, 73^{\circ} 14^{\prime} 59.01^{\prime \prime} \mathrm{E}$ & $50.7 \pm 3.2$ \\
\hline & IS-42 & $33^{\circ} 39^{\prime} 0.44^{\prime \prime} \mathrm{N}, 72^{\circ} 57^{\prime} 57.24^{\prime \prime} \mathrm{E}$ & $21.0 \pm 2.9$ & & NS-92 & $33^{\circ} 39^{\prime} 24.63^{\prime \prime} \mathrm{N}, 73^{\circ} 14^{\prime} 56.93^{\prime \prime} \mathrm{E}$ & $49.8 \pm 2.9$ \\
\hline & IS-43 & $33^{\circ} 38^{\prime} 43.11^{\prime \prime} \mathrm{N}, 72^{\circ} 58^{\prime} 7.96^{\prime \prime E}$ & $30.2 \pm 3.6$ & & NS-93 & $33^{\circ} 39^{\prime} 18.72^{\prime \prime} \mathrm{N}, 73^{\circ} 14^{\prime} 53.66^{\prime \prime} \mathrm{E}$ & $44.9 \pm 3.4$ \\
\hline & IS-44 & $33^{\circ} 38^{\prime} 35.81^{\prime \prime} \mathrm{N}, 72^{\circ} 57^{\prime} 5.25^{\prime \prime} \mathrm{E}$ & $31.4 \pm 3.0$ & & NS-94 & $33^{\circ} 39^{\prime} 14.38^{\prime \prime} \mathrm{N}, 73^{\circ} 14^{\prime} 46.22^{\prime \prime} \mathrm{E}$ & $56.5 \pm 2.9$ \\
\hline & IS-45 & $33^{\circ} 38^{\prime} 5.66^{\prime \prime} \mathrm{N}, 72^{\circ} 55^{\prime} 34.30^{\prime \prime} \mathrm{E}$ & $30.1 \pm 3.7$ & & NS-95 & $33^{\circ} 39^{\prime} 13.10^{\prime \prime} \mathrm{N}, 73^{\circ} 14^{\prime} 38.74^{\prime \prime} \mathrm{E}$ & $51.9 \pm 3.6$ \\
\hline & IS-46 & $33^{\circ} 37^{\prime} 26.86^{\prime \prime} \mathrm{N}, 72^{\circ} 55^{\prime} 27.00^{\prime \prime} \mathrm{E}$ & $29.1 \pm 3.1$ & & NS-96 & $33^{\circ} 39^{\prime} 12.69^{\prime \prime} \mathrm{N}, 73^{\circ} 14^{\prime} 35.32^{\prime \prime} \mathrm{E}$ & $50.1 \pm 3.4$ \\
\hline & IS-47 & $33^{\circ} 40^{\prime} 55.42^{\prime \prime} \mathrm{N}, 73^{\circ} 3^{\prime} 26.07^{\prime \prime} \mathrm{E}$ & $21.8 \pm 2.9$ & & NS-97 & $33^{\circ} 39^{\prime} 11.00^{\prime \prime} \mathrm{N}, 73^{\circ} 14^{\prime} 37.25^{\prime \prime} \mathrm{E}$ & $49.3 \pm 2.8$ \\
\hline & IS-48 & $33^{\circ} 40^{\prime} 15.33^{\prime \prime} \mathrm{N}, 73^{\circ} 2^{\prime} 58.74^{\prime \prime} \mathrm{E}$ & $19.7 \pm 3.1$ & & NS-98 & $33^{\circ} 39^{\prime} 3.30^{\prime \prime} \mathrm{N}, 73^{\circ} 14^{\prime} 30.80^{\prime \prime} \mathrm{E}$ & $47.6 \pm 3.9$ \\
\hline & IS-49 & $33^{\circ} 39^{\prime} 37.31^{\prime \prime} \mathrm{N}, 73^{\circ} 1^{\prime} 28.87^{\prime \prime} \mathrm{E}$ & $16.7 \pm 2.9$ & & NS-99 & $33^{\circ} 38^{\prime} 53.20^{\prime \prime} \mathrm{N}, 73^{\circ} 14^{\prime} 33.00^{\prime \prime} \mathrm{E}$ & $55.8 \pm 2.7$ \\
\hline & IS-50 & $33^{\circ} 38^{\prime} 49.31^{\prime \prime} \mathrm{N}, 73^{\circ} 00^{\prime} 30.13^{\prime \prime E}$ & $23.6 \pm 3.0$ & & NS-100 & $33^{\circ} 38^{\prime} 51.59^{\prime \prime} \mathrm{N}, 73^{\circ} 14^{\prime} 32.62^{\prime \prime} \mathrm{E}$ & $53.7 \pm 3.0$ \\
\hline
\end{tabular}

IS = Soil Samples collected from Islamabad capital territory

NS = Soil samples collected from Nilore

Int. J. Radiat. Res., Vol. 19 No. 2, April 2021 
Table 2. Comparison of present work with reported values of Cesium-137 in literature for various parts of Pakistan and some other countries around the globe.

\begin{tabular}{|c|c|c|c|c|}
\hline Location & $\begin{array}{c}\text { Minimum Activity } \\
(\mathbf{B q} / \mathbf{k g})\end{array}$ & $\begin{array}{c}\text { Maximum Activity } \\
(\mathbf{B k} / \mathbf{k g})\end{array}$ & $\begin{array}{c}\text { Mean values } \\
(\mathbf{B q} / \mathbf{k g})\end{array}$ & References \\
\hline Kohistan, Pakistan & 7.06 & 14.9 & 9.5 & $(35)$ \\
\hline Jhangar Valley, Pakistan & 1.3 & 46.8 & 13.39 & $(38)$ \\
\hline Peshawar and Nowshera, Pakistan & 4.6 & 44.1 & -- & $(39)$ \\
\hline Murree, Pakistan & 1.3 & 54.1 & $13.6 \pm 11.8$ & $(40)$ \\
\hline Jhelum, Pakistan & 0.5 & 4.5 & $2.3 \pm 0.2$ & $(41)$ \\
\hline Attock, Pakistan & 0.4 & 6.7 & $2.4 \pm 0.2$ & $(41)$ \\
\hline Chakwal, Pakistan & 0.8 & 3.7 & $1.7 \pm 0.2$ & $(41)$ \\
\hline Mirpur, Azad Kashmir, Pakistan & 0.076 & 2.94 & 1.39 & $(43)$ \\
\hline Southern Punjab, Pakistan & 0.25 & 3.8 & $1.6+1.1$ & $(42)$ \\
\hline Tehran (Iran) & 0.29 & 28.82 & 11.30 & $(44)$ \\
\hline Tongliao (China) & $0.10 \pm 0.01$ & $21.33 \pm 2.13$ & $4.23 \pm 4.76$ & $(46)$ \\
\hline Bayanwula (China) & 0.2 & $15.6 \pm 1.6$ & $5.64 \pm 0.6$ & $(45)$ \\
\hline Syria & 1.2 & 143 & 37.17 & $(49)$ \\
\hline Ikizdere Valley, Turkey & 3.83 & 6.45 & 5.25 & $(47)$ \\
\hline Louisiana (USA) & 5 & 58 & -- & $(39)$ \\
\hline Vojvodina (Serbia) & 5.7 & 55 & -- & $(48)$ \\
\hline Leningrad Region (Russia) & 29 & 5320 & 467 & $(50)$ \\
\hline Present study & $8.8 \pm 2.8$ & $57.0 \pm 4.2$ & -- & \\
\hline
\end{tabular}

\section{CONCLUSION}

The results of this investigation showed a detectable quantity of Cesium-137 in all collected samples of soil from the Islamabad area.

The highest value for activity concentration of aforementioned nuclide in soil samples collected from a suburb of Islamabad (Nilore) was found to be $57.0 \pm 4.2 \mathrm{~Bq} / \mathrm{kg}$, which is higher than the reported values in the literature for other parts of the country.

The existence of Cesium-137 proved that the studied area is contaminated probably due to fallout from a past nuclear incident that occurred in Ukraine (Chernobyl, 1986) and Japan (Fukushima, 2011) or due to ongoing nuclear activities in this territory.

The detectable level of Cesium-137 in the studied region is insignificant and poses no health risk to the dwellers of this locality; however, concerned local authorities are advised to pay attention to avoid any possible future health hazards occurring to the state population and environment.
This work may also be helpful for providing guidance for further future work regarding evaluation of radioactivity level in the case of the occurrence of any unexpected radiological emergency.

\section{ACKNOWLEDGMENT}

This research work has been supported by the Natural Science Foundation of Heilongjiang Province (ZD2019H003), the Joint Research Fund in Astronomy (U1631239, U1331114) under cooperative agreement between the National Natural Science Foundation of China (NSFC) and Chinese Academy of Sciences (CAS), the International Science \& Technology Cooperation Program of China (2014DFE10030), the 111 project (B13015), the Fundamental Research Funds for the Central Universities to the Harbin Engineering University.

Conflicts of interest: Declared none. 


\section{REFERENCES}

1. Emelue H (2014) Excess Lifetime Cancer Risk due to Gamma Radiation in and Around Warri Refining and Petrochemical Company in Niger Delta, Nigeria. Br J Med Med Res, 4(13): 2590-2598.

2. Qureshi AA, Tariq S, Din KU, Manzoor S, Calligaris C, Waheed A (2014) Evaluation of excessive lifetime cancer risk due to natural radioactivity in the rivers sediments of Northern Pakistan. J Radiat Res Appl Sci, 7(4): 438-447.

3. Ononugbo CP, Avwiri GO, Tutumeni G (2016) Measurement of natural radioactivity and evaluation of radiation hazards in soil of Abua/Odual districts using multivariate statistical approach. Br J Environ Sci, 4(1):35-48.

4. Khan IU, Qin Z, Xie T, Bin Z, Li H, Sun W, Lewis E (2020) Evaluation of health hazards from radionuclides in soil and rocks of North Waziristan, Pakistan. Int J Radiat Res, 18 (2): 243-253.

5. Khan IU, Sun W, Lewis E (2020) Radiological impact on public health from radioactive content in wheat flour available in Pakistani Markets. J Food Prot, 83(2): 377382.

6. Whicker FW, Eisenbud M, Gesell T (1997) Environmental Radioactivity from Natural, Industrial, and Military Sources. Radiat Res, 148(4).

7. Chino M, Nakayama H, Nagai H, Terada H, Katata G, Yamazawa $\mathrm{H}$ (2011) Preliminary Estimation of Release Amounts of 131 I and 137 Cs Accidentally Discharged from the Fukushima Daiichi Nuclear Power Plant into the Atmosphere. J Nucl Sci Technol, 48(7): 1129-1134.

8. Zucchetti M (2013) Radioactivity Transfer in Environment and Food by Fokion K. Vosniakos. Nucl Phys News, 23 (4):39-46.

9. Butkus D, Lukšienè B, Konstantinova M (2009) Evaluation of 137Cs soil-to-plant transfer: Natural and model experiments. J Radioanal Nucl Chem, 279(2): 411-416.

10. UNSCEAR (1993) Sources and effects of ionizing radiation. United Nations Scientific Committee on the Effects of Atomic Radiation. Report to the General Assembly. United Nations, New York.

11. Dabayneh KM, Mashal LA, Hasan FI (2008) Radioactivity concentration in soil samples in the southern part of the West Bank, Palestine. Radiat Prot Dosimetry, 131(2): 265271.

12. Entry JA, Vance NC, Hamilton MA, Zabowski D, Watrud LS, Adriano DC (1996) Phytoremediation of soil contaminated with low concentrations of radionuclides. Water Air Soil Pollut, 88(1): 167-176.

13. Smith GM, Smith KL, Kowe R, Pérez-Sánchez D, Thorne M, Thiry Y, Read D, Molinero J (2014) Recent developments in assessment of long-term radionuclide behavior in the geosphere-biosphere subsystem. J Environ Radioact, 131: 89-109.

14. Steinhauser G, Brandl A, Johnson TE (2014) Comparison of the Chernobyl and Fukushima nuclear accidents: A review of the environmental impacts. Sci Total Environ, 470-471: 800-817.

Int. J. Radiat. Res., Vol. 19 No. 2, April 2021
15. Ferdous J, Begum A, Islam A (2015) Radioactivity of soil at proposed Rooppur Nuclear Power Plant site in Bangladesh. Int J Radiat Res, 13(2): 135-142.

16. Charan Kumar K, Rajendra Prasad T, Narayana Rao T, Venkataratnam M, Nagaraja K (2016) Variation of radon progeny concentration over a continental location. Int J Radiat Res, 14(2):105-111.

17. Tufail M, Sabiha-Javied, Akhtar N, Akhter J (2010) Assessment of annual effective dose from natural radioactivity intake through wheat grain produced in Faisalabad, Pakistan. J Radioanal Nucl Chem, 283(3): 585-590.

18. Abd El-mageed Al, El-Kamel AH, Abbady A, Harb S, Youssef AMM, Saleh II (2011) Assessment of natural and anthropogenic radioactivity levels in rocks and soils in the environments of Juban town in Yemen. Radiat Phys Chem, 80(6): 710-715.

19. Sartandel SJ, Chinnaesakki S, Bara SV, Krishna NS, Vinod Kumar A, Tripathi RM (2014) Assessment of natural and fallout radioactivity in soil samples of Visakhapatnam. J Radioanal Nucl Chem, 299(1): 337-342.

20. Al-Ghamdi H, Al-Muqrin A, El-Sharkawy A (2016) Assessment of natural radioactivity and $137 \mathrm{Cs}$ in some coastal areas of the Saudi Arabian gulf. Mar Pollut Bull, 104(2): 29 $-33$.

21. Baykara O, Doğru M (2010) Assessment of natural radioactivity and heavy metals in water and soil around seismically active area. J Radioanal Nucl Chem, 283(3): 547-553.

22. Okeji MC, Agwu KK, Idigo FU (2012) Assessment of Natural Radioactivity in Phosphate Ore, Phosphogypsum and Soil Samples Around a Phosphate Fertilizer Plant in Nigeria. Bull Environ Contam Toxicol, 89(5): 1078-1081.

23. Penabei $S$, Bongue $D$, Maleka $P$, Dlamini $T$, Saïdou, Guembou Shouop CJ, Halawlaw YI, Ngwa Ebongue A, Kwato Njock MG (2018) Assessment of natural radioactivity levels and the associated radiological hazards in some building materials from Mayo-Kebbi region, Chad. Radioprotection, 53(4): 265-278.

24. Montes ML, Mercader RC, Taylor MA, Runco J, Desimoni J (2012) Assessment of natural radioactivity levels and their relationship with soil characteristics in undisturbed soils of the northeast of Buenos Aires province, Argentina. J Environ Radioact, 105: 30-39.

25. Alnour IA, Wagiran H, Ibrahim N, Hamzah S, Elias MS, Laili Z, Omar M (2014) Assessment of natural radioactivity levels in rocks and their relationships with the geological structure of Johor state, Malaysia. Radiat Prot Dosimetry, 158(2): 201-207.

26. Eštoková A and Palaščáková L (2013) Assessment of Natural Radioactivity Levels of Cements and Cement Composites in the Slovak Republic. Int I Environ Res Public Health, 10(12): 7165-7179.

27. Marović $G$ and Senčar J (1999) Assessment of radioecological situation of a site contaminated by technologically enhanced natural radioactivity in Croatia. J Radioanal NuCl Chem, 241(3): 569-574.

28. Yin N, Lu X, Li Y (2017) Radioactive analysis and radiological hazards of sand in Weifang, China. Int J Radiat Res, 15 (2): 225-228. 
29. Idriss H, Salih I, Alaamer AS, Saleh A, Abdelgali MY (2016) Environmental-Impact Assessment of Natural Radioactivity Around a Traditional Mining Area in Al-Ibedia, Sudan. Arch Environ Contam Toxicol, 70(4): 783-792.

30. Kobeissi MA, El-Samad O, Rachidi I (2013) Health assessment of natural radioactivity and radon exhalation rate in granites used as buildingmaterials in Lebanon. Radiat Prot Dosimetry, 153(3): 342-351.

31. Al-Kharouf SJ, Al-Hamarneh IF, Dababneh M (2008) Natural radioactivity, dose assessment and uranium uptake by agricultural crops at Khan Al-Zabeeb, Jordan. J Environ Radioact, 99(7): 1192-1199.

32. González-Fernández D, Garrido-Pérez MC, Casas-Ruiz M, Barbero L, Nebot-Sanz E (2012) Radiological risk assessment of naturally occurring radioactive materials in marine sediments and its application in industrialized coastal areas: Bay of Algeciras, Spain. Environ Earth Sci, 66(4): 1175-1181.

33. Isinkaye MO (2008) Radiometric assessment of natural radioactivity levels of bituminous soil in Agbabu, southwest Nigeria. Radiat Meas, 43(1): 125-128.

34. Taheri A, Taheri A, Fathivand AA, Mansouri N (2019) Risk assessment of naturally occurring radioactive materials (NORM) in the hydrocarbon sludge extracted from the south pars gas field in Iran. Process Saf Environ Prot, 125: 102-120.

35. IAEA (1989) Measurement of Radionuclides in Food and the Environment. International Atomic Energy Agency, Vienna.

36. IAEA (2000) Radionuclides and trace elements in soil. Reference Material IAEA-375. Analytical Quality Control Services (AQCS) of International Atomic Energy Agency (IAEA), Vienna, Austria.

37. Khan HM, Ismail M, Khan K, Akhter P (2011) Radioactivity Levels and Gamma-Ray Dose Rate in Soil Samples from Kohistan (Pakistan) Using Gamma-Ray Spectrometry. Chinese Phys Lett, 28(1): 019301.

38. Khan HM, Chaudhry ZS, Ismail M, Khan K (2010) Assessment of Radionuclides, Trace Metals and Radionuclide Transfer from Soil to Food of Jhangar Valley (Pakistan) Using Gamma-Ray Spectrometry. Water, Air and Soil Pollut, 213(1-4): 353-362.

39. Ismail M, Zia MA, Khan HM (2017) Investigation of Cs-137 in the environmental soil segments of the Peshawar and Nowshera districts of Khyber Pakhtunkhwa, Pakistan. Int J Radiat Res, 15(4): 407-412.

40. Khizar Hayat S, Jabbar T, Dilband M, Khalid K, Rashid A,
Jabbar A (2017) Assessment of background radiation levels and associated doses in soils of the most popular tourist place Muree, Pakistan. Univers J Eng Sci, 5(4): 64-69.

41. Rahman SU, Matiullah, Malik F, Rafique M, Anwar J, Ziafat M, Jabbar A (2011) Measurement of naturally occurring/ fallout radioactive elements and assessment of annual effective dose in soil samples collected from four districts of the Punjab Province, Pakistan. J Radioanal Nucl Chem, 287(2): 647-655.

42. Fatima I, Zaidi JH, Arif M, Daud M, Ahmad SA, Tahir SNA (2008) Measurement of natural radioactivity and dose rate assessment of terrestrial gamma radiation in the soil of southern Punjab, Pakistan. Radiat Prot Dosimetry, 128(2): 206-212.

43. Rafique M (2014) Cesium-137 activity concentrations in soil and brick samples of Mirpur, Azad Kashmir Pakistan. Int J Radiat Res, 12(1): 39-46.

44. Osouli A, Abbasi F, Naseri M (2009) Measurement of ${ }^{137} \mathrm{Cs}$ in soils of Tehran province. Iran J Radiat Res, 7(3): 141149.

45. Bai H, Hu B, Wang C, Bao S, Sai G, Xu X, Zhang S, Li Y (2017) Assessment of radioactive materials and heavy metals in the Surface soil around the Bayanwula Prospective Uranium mining area in China. Int J Environ Res Public Health, 14(3): 300-307.

46. Haribala, Hu B, Wang C, Gerilemandahu, Xu X, Zhang S, Bao S, Li Y (2016) Assessment of radioactive materials and heavy metals in the surface soil around uranium mining area of Tongliao, China. Ecotoxicol Environ Saf, 130: 185192.

47. Keser R, Korkmaz Görür F, Alp I, Okumuşoğlu NT (2013) Determination of radioactivity levels and hazards of sediment and rock samples in ikizdere and Kaptanpaşa Valley, Turkey. Int J Radiat. Res,11(3): 155-165.

48. Forkapic S, Vasin J, Bikit I, Mrdja D, Bikit K, Milić S (2017) Correlations between soil characteristics and radioactivity content of Vojvodina soil. J Environ Radioact, 166: 104111.

49. Al-Masri MS, Amin Y, Hassan M, Ibrahim S (2006) External gamma-radiation dose to Syrian population based on the measurement of gamma-emitters in soils. J Radioanal Nucl Chem, 267(2): 337-343.

50. Ylipieti J, Rissanen K, Kostiainen E, Salminer R, Tomilina O, Tahk K, Gilucis A, Gregorauskiene V (2008) Chernobyl fallout in the uppermost $(0-3 \mathrm{~cm})$ humus layer of forest soil in Finland, North East Russia and the Baltic countries in 2000-2003. Sci Total Environ, 407(1): 315-323. 